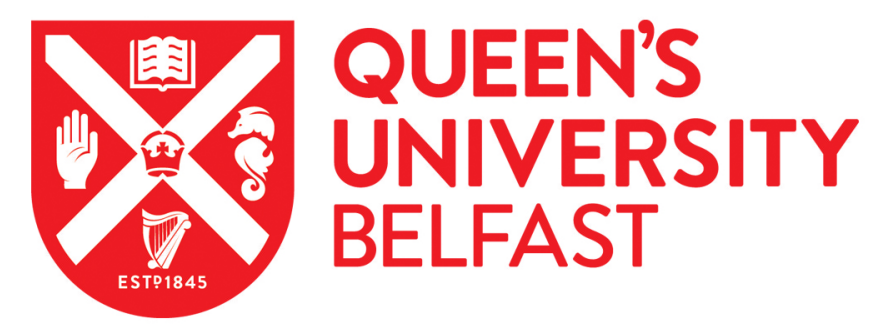

\title{
A novel RCE1 isoform is required for H-Ras plasma membrane localization and is regulated by USP 17
}

Jaworski, J., Govender, U., McFarlane, C., De La Vega, M., Greene, M. K., Rawlings, N. D., Johnston, J. A., Scott, C. J., \& Burrows, J. F. (2014). A novel RCE1 isoform is required for H-Ras plasma membrane localization and is regulated by USP17. Biochemical Journal, 457(2), 289-300. https://doi.org/10.1042/BJ20131213

Published in:

Biochemical Journal

Document Version:

Peer reviewed version

Queen's University Belfast - Research Portal:

Link to publication record in Queen's University Belfast Research Portal

Publisher rights

(C) 2014 Biochemical Journal

This is no the final peer-reviewed Version of Record

\section{General rights}

Copyright for the publications made accessible via the Queen's University Belfast Research Portal is retained by the author(s) and / or other copyright owners and it is a condition of accessing these publications that users recognise and abide by the legal requirements associated with these rights.

Take down policy

The Research Portal is Queen's institutional repository that provides access to Queen's research output. Every effort has been made to ensure that content in the Research Portal does not infringe any person's rights, or applicable UK laws. If you discover content in the Research Portal that you believe breaches copyright or violates any law, please contact openaccess@qub.ac.uk. 


\title{
A novel RCE1 isoform is required for $\mathrm{H}$-Ras plasma membrane localisation and is regulated by USP17
}

\author{
Jakub Jaworski*, Ureshnie Govender ${ }^{*}{ }^{*}$, Cheryl McFarlane ${ }^{\dagger}$, Michelle de la Vega $^{\dagger}$, Michelle K. \\ Greene $^{*}$, Neil D. Rawlings ${ }^{\ddagger} \S^{\S}$ James A. Johnston ${ }^{\dagger}$," , Christopher J. Scott ${ }^{*}$, James F. Burrows $^{*, 1}$ \\ * School of Pharmacy, Queen's University Belfast, McClay Research Building, 97 Lisburn Road, \\ Belfast, BT9 7BL, UK. † Centre for Infection and Immunity, School of Medicine, Dentistry and \\ Biomedical Sciences, Queen's University Belfast, Health Sciences Building, 97 Lisburn Road, \\ Belfast, BT9 7BL, UK. $\ddagger$ Wellcome Trust Sanger Institute, Cambridge CB10 1SA, UK. § European \\ Molecular Biology Laboratory, European Bioinformatics Institute, Wellcome Trust Genome Campus, \\ Hinxton, CB10 1SD, UK. 11 Current address; Inflammation Research, Amgen Inc., One Amgen \\ Center Dr., Thousand Oaks, CA 91320, USA.
}

Processing of the ' $\mathrm{CaaX}$ ' motif found on the carboxy termini of many proteins, including the protooncogene Ras, requires the ER resident protease Ras converting enzyme 1 (RCE1) and is necessary for the proper localisation and function of many of these ' $\mathrm{CaaX}$ ' proteins. Here we report that several mammalian species have a novel isoform (Isoform 2) of RCE1 resulting from an alternate splice site and producing an amino terminally truncated protein. We demonstrate that both RCE1 isoform 1 and the newly identified isoform 2 are required to reinstate proper $\mathrm{H}$-Ras processing and thus plasma membrane localisation in RCE1 null cells. In addition, we show that the deubiquitinating enzyme USP17, previously shown to modulate RCE1 activity, can regulate the abundance and localisation of isoform 2. Furthermore, we show that isoform 2 is ubiquitinated on lysine 43 and deubiquitinated by USP17. Collectively, these findings indicate that RCE1 isoform 2 is required for proper ' $\mathrm{CaaX}$ ' processing and that USP17 can regulate this via its modulation of RCE1 isoform 2 ubiquitination.

Key words: Deubiquitinating, Protease, Ras, RCE1, Ubiquitin, USP17

\section{INTRODUCTION}

Many proteins, including members of the Ras small GTPase super family, contain a 'CaaX' motif (C: cysteine, a: aliphatic residue, $\mathrm{X}$ : any amino acid) at their carboxy termini. This motif, best characterised in the proto-oncogene Ras, undergoes a number of sequential post-translational modifications, which in the case of Ras, facilitates its re-localisation to the plasma membrane. Briefly, the cysteine residue is first isoprenylated by a farnesyltransferase (FTase) or a geranylgeranyltransferase (GGTase) [1] and then Ras is localized to the endoplasmic reticulum (ER) where the three carboxy terminal amino acids (i.e., the -aaX) are removed by Ras converting enzyme 1 (RCE1), an ER integral trans-membrane protease [2, 3, 4]. Subsequently, the exposed isoprenylcysteine is methylated by a trans-membrane methyltransferase called isoprenylcysteine carboxyl methyltransferase (ICMT) [5]. Finally, depending on the Ras isoform, it may then be palmitoylated $[6,7]$ prior to plasma membrane trafficking.

$\mathrm{RCE} 1$, has been reported to be vital for the membrane localisation of the $\mathrm{H}$ - and $\mathrm{N}$ - isoforms of Ras, whilst K-Ras-4b would appear, at least partially, to localise to the plasma membrane in its absence [8]. Little is known about the catalytic mechanism or regulation of RCE1. It had initially been identified as a cysteine protease, but subsequent studies would suggest that it has metalloprotease-like activity, or represents a novel class of proteolytic enzyme $[9,10]$. Also, until our recent work showing that the deubiquitinating enzyme USP17 can modulate RCE1 activity [11] nothing was known about the regulation of RCE1, although it had been shown that Ras processing can be regulated by growth

1 To whom correspondence should be addressed. Email: j.burrows@qub.ac.uk 
factor-induced phosphorylation of the $\alpha$ and $\beta$-subunits of FTase [12, 13, 14].

The DUB/USP17 subfamily of deubiquitinating enzymes were originally identified as murine (DUB-1, DUB-1A, DUB-2) and human (USP17) cytokine inducible genes [15, 16, 17] and a number of family members have been shown by us, and others, to regulate cell growth, survival and movement $[11,16,17,18,19,20,21]$. In particular, we and others have shown that constitutive expression of USP17 can block cell proliferation and that tight regulation of USP17 expression is necessary for cell cycle progression [11, 17, 20,21, 22]. In addition, we have shown that USP17 is required for chemokine driven cell migration [23]. More recently we have demonstrated that USP17 disrupts $\mathrm{H}$ - and $\mathrm{N}$-Ras plasma membrane localisation and activation $[11,24]$ and that these effects are due to the attenuation of Ras processing; a result of the inhibition of RCE1 by USP17 [11].

In this current study we have further analysed RCE1 and its regulation. We show that RCE1 has an additional novel isoform which is produced due to the utilization of an alternative splice site and results in an amino terminally truncated protein. We also demonstrate that this novel isoform is required to reconstitute H-Ras plasma membrane localisation in RCE1-/- mouse embryonic fibroblast (MEF) cells and that its abundance and localisation are regulated by USP17. In addition, we also show that this novel isoform is ubiquitinated upon lysine 43 using lysine 63 linked ubiquitin chains, and that this ubiquitin can be removed by USP17. Taken together, these findings indicate this novel isoform is necessary for ' $\mathrm{CaaX}$ ' processing and that by modulating the abundance and location of this novel isoform, via its deubiquitination, USP17 can regulate RCE1 activity.

\section{EXPERIMENTAL}

\section{Plasmids}

The pDQ-EV (His), pDQ-USP17 (His), and pDQ-USP17CS (His) were kind gifts from Dr. Derek Quinn (Queen's University, Belfast). The HA-ubiquitin, GFP-H-Ras, USP17 shRNA1, USP17 shRNA2 and the scrambled shRNA were described previously [11, 22, 23]. RCE1 isoform 1 and isoform 2 cDNAs were tagged with the FLAG epitope at their C-termini as described previously [11]. All mutants were produced using a site directed mutagenesis kit as per the instructions (Stratagene, USA). In order to generate His tagged isoforms of RCE1 (RCE1-ISO1-HIS; RCE1-ISO2-HIS) the coding sequences were sub-cloned into the $\mathrm{pDQ}$ vector as a fusion protein with a C-terminal His6 Tag spaced by a short linker (GSG).

\section{Cell Culture and DNA Transfections}

293T and MEF cells were cultured as described previously [11]. HeLa cells (American Type Culture Collection (ATCC), Manassas, USA) were grown in DMEM supplemented with 10\% FCS (PAA), $1 \%$ penicillin $(10,000 \mathrm{U} / \mathrm{ml}) /$ streptomycin $(10,000 \mu \mathrm{g} / \mathrm{ml})$, and $1 \%$ L-glutamine $(200 \mathrm{mM})$. Cells lines were all grown at $37^{\circ} \mathrm{C}$ in a $5 \% \mathrm{CO}_{2}$ humidified incubator. Cells were transfected with FuGENE $^{\mathrm{TM}} 6$ transfection reagent (Roche) according to manufacturer's instructions. Cells were seeded between $0.5 \times 10^{6}$ and $1.0 \times 10^{6}$ cells for protein experiments or 0.7-2.5 × $10^{4}$ on 4-well glass culture slides (BD Falcon) for microscopy experiments. The cells were transfected with $2 \mu \mathrm{g}$ of plasmid DNA for protein experiments and biological assays or $0.25 \mu \mathrm{g}$ of plasmid DNA for confocal microscopy experiments.

\section{RNA extraction and reverse transcription-PCR}

RNA was extracted using STAT-60 (Biogenesis) according to the manufacturer's instructions. Reverse transcription-PCR (RT-PCR) was performed on $1 \mu \mathrm{g}$ of total RNA using OneStep RT-PCR kit (Qiagen) as described previously [22]. The following primers were used: USP17, 5'-

CAGTGAATTCGTGGGAATGGAGGACGACTCAC TCTAC-3' (forward) and 5'AGTCATCGATCTGGCACACAAGCATAGCCCTC-3' (reverse). GAPDH, 5'ATGGCAAATTCCATGGCA-3' (forward) and 5'-TCTAGACGGCAGGTCAGG-3' (reverse). RCE1 isoform 1, 5'-TGCTGGGTGTCAGTGTTCTC-3' (forward) and 5'CAGGGATGTGCCTGGCTGGA-3' (reverse). RCE1 isoform 2, 5'-TGCTGGGTGTC AGTGT 
TCTC-3' (forward) and 5'-CAGGGATGTGCCTGGATGCC-3' (reverse). Beta actin, 5'-

GGACTTCGAGCAAGAGATGG-3' (forward) and 5'-AGCACTGTGTTGGCGTACAG-3' (reverse).

\section{Cell Lysis and Immunoblotting}

Cells were lysed in the following buffer: $25 \mathrm{mM}$ TrisHCl pH 7.6, $150 \mathrm{mM} \mathrm{NaCl}, 1 \% \mathrm{NP}-40,1 \%$ sodium deoxycholate, $0.1 \%$ SDS, supplemented with protease inhibitor cocktail complete mini (Roche, Germany). Lysates were left on ice for 20mins before being centrifuged at 15,000xg for $10 \mathrm{mins}$ at $4^{\circ} \mathrm{C}$. Equal volumes of whole cell lysate were added to Laemmli buffer to a final concentration of $1 \mathrm{X}$ with $5 \% \beta$-mercaptoethanol. The samples were boiled for $5 \mathrm{~min}$ at $99^{\circ} \mathrm{C}$ for protein denaturation. The samples were analyzed by SDS-PAGE and Western blotting on PVDF membrane (Millipore, UK). The membranes were then blocked in 5\% marvel in 0.1\% Tween-20/TBS for $1 \mathrm{hr}$. After blocking, the membranes were probed with the indicated antibodies for $1 \mathrm{hr}$ at RT or overnight at $4^{\circ} \mathrm{C}$. The following primary antibodies were used: rat anti-tubulin (Abcam, UK), antiFLAG (Sigma, UK), rabbit polyclonal anti-DUB-3/USP17 (Fusion Antibodies, Belfast, UK), anti-HA (Covance, USA). The membrane was incubated with the appropriate secondary antibody: either goat anti-mouse HRP conjugate (Bio-Rad, USA), goat anti-rabbit HRP conjugate (Bio-Rad) or rabbit antirat HRP conjugate (Abcam). Proteins were detected with a chemiluminescence protocol and were exposed using the ChemiDoc XRS imaging system (BioRad).

In order to pull down proteins tagged with C-terminal HisTag in denaturing conditions immobilized-metal affinity chromatography was used. RIPA whole cell lysates were prepared as above. IMAC resin was prepared as follows: $1 \mathrm{~mL}$ of IMAC Sepharose 6 Fast Flow resin (GE Healthcare, USA) was washed in $10 \mathrm{~mL}$ of deionized water twice. The resin was charged with metal ion by resuspension in $50 \mathrm{mM} \mathrm{NiSO}_{4}$ (Sigma). An excess of metal ions was removed by subsequent two $10 \mathrm{~mL}$ washes with deionized water. Finally nickel charged resin was resuspended in $10 \mathrm{~mL}$ Denaturation Buffer ( $8 \mathrm{M}$ urea, $300 \mathrm{mM} \mathrm{NaCl}, 0.1 \%$ SDS, $50 \mathrm{mM}$ sodium monobasic phosphate, $5 \mathrm{mM}$ imidasole, $1 \mathrm{mM} \beta$-mercaptoethanol, $\mathrm{pH}=8.0$ ). Subsequently, magnesium chloride (Sigma) was added to clarified whole cell lysates in RIPA buffer to a final concentration of $5 \mathrm{mM}$. Immediately after $200 \mu \mathrm{L}$ of cell lysate was mixed with $1 \mathrm{~mL}$ of resuspended resin and incubated for $1 \mathrm{hr}$ at RT with gentle agitation. Following incubation the resin was pelleted $(1000 \mathrm{~g}, 1 \mathrm{~min}, \mathrm{RT})$ and washed six times in $1 \mathrm{~mL}$ of Denaturation Buffer before being resuspended in an appropriate volume of 2x Laemmli Buffer.

\section{Confocal Microscopy}

HeLa and MEF cells were seeded at $0.7-2.5 \times 10^{4}$ cells $/ 1.7 \mathrm{~cm}^{2}$ well of glass culture slides (BD Falcon). Cells were transfected as previously described. The cells were fixed in $4 \%$ parafomaldehyde in PBS for 20mins. The cells were then permeabilized in 0.5\% Triton X-100 in PBS for 5mins, washed in PBS and blocked in blocking solution (1\% BSA, 10\% donkey serum in PBS) for $1 \mathrm{hr}$ at RT. Transfected proteins and cell organelles were stained with appropriate antibodies or counter stains according to manufacturer's protocol. Antibodies and co-stains were as follows: DAPI (Invitrogen, USA), anti-FLAG-FITC (Sigma), mouse monoclonal anti-DUB-3/USP17 (Fusion Antibodies), antiHA (Roche), anti-calnexin (Jackson Laboratories, USA), donkey anti-mouse or rabbit Cy5 (Jackson ImmunoResearch), donkey anti-mouse or rabbit TRITC (Jackson ImmunoResearch), donkey antimouse Alexa Fluor 488 (Invitrogen) or donkey anti-rabbit Alexa Fluor 568 (Invitrogen). The slides were sealed with a coverslip and Prolong Gold Antifade mounting media (Invitrogen). Slides were viewed on a Leica SP5 Confocal Microscope. Fluorescent images were captured with a 63x lens zoomed 1-4x with a 1024 x 1024 frame and $400 \mathrm{~Hz}$ scanning speed. Images were analyzed using Leica LAS AF software and Fiji. The images presented in the same figures were captured using standardized setting and exposure times. 


\section{RESULTS}

\section{There are two mammalian RCE1 transcripts}

Whilst examining the sequence databases we observed that two human transcripts for RCE1 were annotated within the RCE1 gene record (Isoform 1 [GenBank: NM_005133] and Isoform 2 [GenBank: NM_001032279]) (Figure 1a and Supplemental Figure 1). Isoform 1 represents the full length protein that has been reported previously in the literature and has been the subject of our, and others, investigations into this enzyme to date. The second transcript differs due to the utilisation of an alternative splice site between exons 2 and 3 (Figure 1a and Supplemental Figure 1) which leads to the loss of four base pairs (CCAG) and results in an $\mathrm{N}$ terminally truncated protein, initiating at methionine 105 (numbering based on RCE1 isoform 1) (Figure 1a and Supplemental Figure 1). This novel isoform has not previously been reported, but further analysis revealed that transcripts equivalent to isoforms 1 and 2 are annotated in Mouse (Mus musculus) (Supplemental Figure 2, Isoform 1 [GenBank: NM_023131] and Isoform 2 [GenBank: BC004060]), Chimpanzee (Pan troglodytes)(Supplemental Figure 3, Isoform 1 [GenBank: GABE01003899] and Isoform 2 [GenBank: GABE01015691]), Rhesus monkey (Macaca mulatta)(Supplemental Figure 4, Isoform 1 [GenBank: JV667132] and Isoform 2 [GenBank: JV728199]) and Goat (Capra hircus)(Supplemental Figure 5, Isoform 1 [GenBank: JO595975] and Isoform 2 [GenBank: KA346662]) suggesting both are bona fide RCE1 isoforms which are potentially conserved throughout a range of mammalian species. To further confirm the presence of both RCE1 transcripts we designed RT-PCR primer sets which specifically recognise either human RCE1 isoform 1, or RCE1 isoform 2, and confirmed their presence in mRNA from a number of human cell lines (Figure 1b).

Endogenous RCE1 cannot be detected due to the lack of a reliable antibody, but previously we had produced an expression vector for RCE1 isoform 1 with a carboxy terminal FLAG epitope tag (RCE1-ISO1-FLAG) and we were able to confirm the stable expression of this protein by Western blotting [11]. As before, we constructed an equivalent expression construct for RCE1 isoform 2 (RCE1-ISO2-FLAG) and then transfected both expression vectors into 293T cells and assessed their relative expression by Western blotting (Figure 1c). Bands of approximately the expected sizes (Isoform 1; $30 \mathrm{KDa}$, Isoform 2; $25 \mathrm{KDa}$ ) were observed for both isoforms with isoform 2 appearing to be the more abundant protein (Figure 1c). In addition, a lower molecular weight band ( 23KDa) was visualised upon the expression of isoform 2 and this appeared to be the predominant form of isoform 2 observed (Figure 1c). This suggested that both isoforms could produce stable proteins and that isoform 2 potentially undergoes post-translational processing.

\section{RCE1 isoform 2 is regulated by USP17}

Our previous studies had indicated that USP17 could regulate RCE1 activity by removing ubiquitin from this protease [11], although the exact nature of this regulation was unclear. The data at this point now suggested that isoform 2 was a conserved bona fide transcript of RCE1 which could produce a stable protein. Therefore, we examined the impact of USP17 upon RCE1 isoform 2 to see if it could play any role in this regulation. We co-transfected 293T cells with combinations of expression constructs for RCE1 isoform 2, USP17 and USP17CS (catalytically inactive mutant) and observed that co-expression of USP17 dramatically reduced the abundance of RCE1 isoform 2, whilst USP17CS had no impact (Figure 2a). We then co-expressed isoform 2 with a control shRNA and two previously characterised USP17 specific shRNAs [22, 23] and demonstrated that USP17 knockdown markedly increased the level of RCE1 isoform 2 (Figure 2b). This was in contrast to what we observed for RCE1 isoform 1 which was unaffected by USP17 (Supplemental Figure 6a).

This indicated that USP17 could modulate the abundance of RCE1 isoform 2 and to examine if this was due to a direct deubiquitination we co-expressed RCE1-ISO2-HIS and HA-ubiquitin with either USP17 or USP17CS, in 293T cells. Upon lysis each sample was incubated with IMAC beads under denaturing conditions to allow the capture of the HIS-tagged RCE1 isoform 2 before the lysates and precipitates were assessed by Western blotting (Figure 2c). Upon blotting with an antibody against HA-ubiquitin we observed a distinctive ladder like pattern equivalent to the addition of at least one, two and three ubiquitin molecules to the previously observed RCE1 isoform 2 bands (Figure 2c). 
More interestingly, in the presence of USP17, but not USP17CS, these bands were absent (Figure 2c, lane 2) indicating that RCE1 isoform 2 was indeed ubiquitinated and that USP17 could remove this ubiquitin.

The observation that USP17 deubiquitinated RCE1 isoform 2, but lead to a drop in its abundance, suggested that isoform 2 ubiquitination was not simply targeting it to the proteasome. This was further supported by experiments demonstrating that the ubiquitin chains utilised were lysine 63 linked. In particular, expression of a ubiquitin mutant lacking lysine 63 (K63R) resulted in nearly a complete loss of RCE1 isoform 2 ubiquitination. Conversely, expression of a mutant with no lysine residues other than 63 (R63K) led to a ubiquitination pattern identical to that of wild-type ubiquitin (Supplemental Figure 6b).

This now indicated that RCE1 isoform 2 ubiquitination was not directly targeting it for degradation and we further investigated what role it may play. We examined the localisation of isoform 2 to determine if it was restricted to the endoplasmic reticulum (ER) as RCE1 isoform 1 had been shown to localise to the ER in both yeast [25] and mammalian cells [11, 22]. Hela cells were transfected with RCE1-ISO2-FLAG in combination with an empty vector or an expression constructs for either USP17 or USP17CS, stained using an anti-calnexin antibody as a marker for the ER, and an anti-FLAG antibody before being assessed using confocal microscopy (Figure 2d). A peri-nuclear localisation, which strongly overlapped with the ER marker calnexin, was observed for RCE1 isoform 2 when it was expressed on its own or in the presence of USP17CS (Figure 2d, bottom panels 1 and $3)$. However, in the presence of USP17 there was a significant reduction in the co-localisation of RCE1 isoform 2 with calnexin (Figure 2d, bottom panel 2, Supplemental Figure 6c). When RCE1 isoform 1 was similarly examined, USP17 had no impact upon its localisation (Figure 2d, top panels, Supplemental Figure 6c). These results demonstrated that USP17 could modulate the localisation of RCE1 isoform 2 and indicated that this may account for its previously demonstrated regulation of RCE1 activity [11].

\section{RCE1 isoform 2 over-expression can reverse the re-localisation of H-Ras by USP17}

Our results to date indicated USP17 could regulate 'CaaX' processing via the modulation of RCE1 isoform 2 levels and/or location. To further assess if this was the case we transfected Hela cells with combinations of expression constructs for GFP-H-Ras, USP17, USP17CS and RCE1-ISO2-FLAG and assessed the localisation of GFP-H-Ras to determine the activity of RCE1. When GFP-H-Ras was transfected alone, or in conjunction with USP17CS, plasma membrane localisation was predominantly observed (Figure 3a, left panels top and bottom). As previously reported [11], when co-expressed with USP17, GFP-H-Ras localisation shifted to the cytosol or internal structures, due to the loss of proper 'CaaX' processing (Figure 3a, left panel middle). However, when RCE1-ISO2-FLAG was also cotransfected, GFP-H-Ras appeared to remain mainly localised to the plasma membrane even in the presence of USP17 expression (Figure 3a, right panel, top, middle and bottom). This was further confirmed and quantified by scoring 100 cells in each condition for either predominantly plasma membrane (PM) or cytosolic (cytosol) distribution and the results of three separate experiments are illustrated (Figure 3b). This confirmed the clear shift of GFP-H-Ras to the cytosol in the presence of USP17, and that this shift was blocked by the over-expression of RCE1 isoform 2.

\section{Both RCE1 isoforms are required to reconstitute H-Ras plasma membrane localisation in RCE1-/- cells}

The demonstration that RCE1 isoform 2 was degraded upon USP17 expression and that its overexpression could block the impact of USP17 upon H-Ras localisation, suggested that isoform 2 is necessary for H-Ras plasma membrane localisation. To examine this, we examined which RCE1 isoforms were necessary to reconstitute H-Ras plasma membrane localisation in RCE1-/- mouse embryonic fibroblast (MEF) cells. Wild-type (WT) and RCE1-/- MEF cells were transfected with combinations of expression constructs for GFP-H-Ras, RCE1-ISO1-FLAG and RCE1-ISO2-FLAG. As expected, in WT MEF cells GFP-H-Ras showed a marked plasma membrane localisation (Figure 4a, left panel). Alternatively, when transfected into RCE1-/- MEF cells, GFP-H-Ras was predominantly observed in the cytosol, or upon internal cellular structures, and this localisation was 
not altered by the expression of either RCE1 isoform 1 or isoform 2 alone (Figure 4a, middle panels). However, when both RCE1 isoform 1 and 2 were expressed together, GFP-H-Ras now strongly localised to the plasma membrane (Figure 4a, right panels). In addition, those RCE1-/- MEF cells transfected with both RCE1 isoforms also appeared to undergo a change in morphology, with a generally more elongated, fibroblastic-like shape being predominantly observed (Figure 4a, right panels). To further confirm and quantify these observations, we scored 100 cells in each condition for either predominantly plasma membrane (PM) or cytosolic (cytosol) distribution and the results of three independent experiments are illustrated (Figure 4b). This confirmed the clear shift of GFP-HRas to the plasma membrane in RCE1-/- MEF cells transfected with both RCE1 isoforms and indicated that both RCE1 isoform 1 and RCE1 isoform 2 are essential for the localisation of H-Ras to the plasma membrane.

\section{Lysine 43 (K43) is required for the proper localisation and function of RCE1 isoform 2}

Next, we examined the molecular basis for the regulation of RCE1 isoform 2 by USP17. To confirm the importance of RCE1 isoform 2 ubiquitination we now examined the importance of its lysine residues, and thus its ubiquitination, in regards to H-Ras localisation. To do this we again transfected constructs for GFP-H-Ras and various combinations of RCE1 isoforms 1 and 2 and equivalent mutants lacking any lysine residues (Isoform 1; K57R, K69R, K147R, K306R: Isoform 2; K43R, K202R) into RCE1-/- MEF cells and examined the localisation of H-Ras by confocal microscopy. The isoform 1 mutant lacking lysine residues was still able to reconstitute H-Ras plasma localisation when co-expressed with wild-type isoform 2 (Figure 5a, middle left panels). However, when the isoform 2 mutant lacking lysine residues was used in these experiments, it could not reconstitute H-Ras plasma membrane localisation, indicating it was no longer active (Figure 5a, middle right and right panels). We confirmed and quantified these observations by scoring 100 cells in each condition for a plasma membrane or cytosolic distribution and these results are illustrated in Figure 5b.

This now indicated that the ubiquitination of isoform 2 was crucial to its function and we further investigated as to which lysine residue was involved. To do this we repeated our previous experiments using mutants of isoform 2 lacking the individual lysine residues (K43R, K202R). The isoform 2 mutant lacking lysine 202, in combination with isoform 1, could reconstitute H-Ras plasma membrane localisation (Figure 6a, right panels). However, the isoform 2 mutant lacking lysine 43, even in conjunction with isoform 1, could not localise H-Ras to the plasma membrane (Figure 6a, middle panels), something which was confirmed and quantified by scoring 100 cells in each condition for plasma membrane or cytosolic distribution (Figure 6b).

This now indicated that lysine 43 of RCE1 isoform 2 was the crucial ubiquitination site and that USP17 could regulate isoform 2 by deubiquitinating lysine 43 . To further confirm this ubiquitination we co-expressed, RCE1-ISO2-HIS, RCE1-ISO2-K43R-HIS, or RCE1-ISO2-K202RHIS with HA-Ubiquitin in 293T cells and upon lysis we again incubated them with IMAC beads under denaturing conditions to allow the precipitation of these HIS-tagged proteins prior to assessment by Western blotting (Figure 6c). Upon blotting with an antibody against HA-ubiquitin we again observed a ladder like pattern equivalent to the addition of various multiples of ubiquitin molecules to both the wild-type and K202R mutant of RCE1 isoform 2 (Figure 6c, lanes 1 and 3). However, no such bands were observed for the K43R mutant of RCE1 isoform 2, even though the presence of a roughly equivalent amount of protein was observed when the precipitates were blotted with an anti-HIS antibody (Figure 6c, lane 2). This indicated that RCE1 isoform 2 was indeed ubiquitinated on lysine 43.

To further confirm if this ubiquitination was important for the localisation of RCE1 isoform 2 we again transfected Hela cells with either RCE1-ISO2-K43R-FLAG or RCE1-ISO2-K202R-FLAG alone, or in combination with either USP17 or USP17CS, and stained the resulting slides using an anti-calnexin antibody as a marker for the ER, and an anti-FLAG antibody before assessing them via confocal microscopy (Figure 7a). Again, a peri-nuclear localisation, which strongly co-localised with the ER marker calnexin, was observed for the RCE1 isoform $2 \mathrm{~K} 202 \mathrm{R}$ mutant when it was cotransfected with either empty vector or an expression construct for USP17CS (Figure 7a, bottom panels 1 and 3). Also, as observed for wild-type isoform 2, in the presence of USP17 a significant 
proportion of RCE1 isoform $2 \mathrm{~K} 202 \mathrm{R}$ mutant did not overlap with the ER marker (Figure 7a, bottom panel 2, Supplemental Figure 7a). However, when the RCE1 isoform 2 K43R mutant was examined, it did not co-localise strongly with the ER marker whether or not USP17 was expressed (Figure 7a, top panels, Supplemental Figure 7a). These results indicated that the ubiquitination of isoform 2 is important for its localisation to the ER and further supported that USP17 could regulate the localisation of RCE1 isoform 2 through its deubiquitination.

USP17 had also been shown to impact upon the stability of RCE1 isoform 2 suggesting the ubiquitination of RCE1 isoform 2 lysine 43 can impact upon the stability of this protein. To further investigate this we now co-expressed RCE1 isoform 2 and the two individual lysine mutants with either USP17 or USP17CS in 293T cells and observed, as before (Figure 2a), that co-expression of USP17 dramatically reduced the abundance of wild-type isoform 2 and the isoform 2 K202R mutant, whilst USP17CS had no impact (Figure 7b, Supplemental Figure 7b). Unexpectedly, the loss of lysine 43 didn't appear to impact upon the stability of RCE1 isoform 2, as comparable amounts of protein were observed for this mutant and wild-type RCE1 isoform 2 (Figure 7b, Supplemental Figure 7b). However, USP17 was unable to influence the abundance of the K43R mutant (Figure 7b, Supplemental Figure 7b) indicating its regulation of RCE1 isoform 2 abundance requires lysine 43 ubiquitination.

\section{USP17 impact upon cell proliferation is RCE1 Isoform 2 dependent}

This study indicates that RCE1 isoform 2 is a substrate for USP17. However, other substrates have been proposed for USP17 including Cdc25A [21] and SDS3 [26]. Previously we have shown that USP17 could not impact upon the growth of cells lacking RCE1 [11] suggesting RCE1 is a major USP17 substrate. Therefore, we now wanted to determine if RCE1 isoform 2 is the USP17 substrate responsible for this impact upon growth. Here we have demonstrated that USP17 knockdown stabilises RCE1 isoform 2 and previously we have shown that USP17 knockdown blocks cell growth [22]. Therefore, we examined if RCE1 isoform 2 over-expression could impact upon cell growth. To accomplish this we transfected Hela and 293T cells with USP17shRNA1, RCE1-ISO2-FLAG or RCE1-ISO2-K43R-FLAG and compared the growth of these transfected cells to mock transfected control cells (Figure 7c, Supplemental Figure 8). As observed previously, USP17 knockdown dramatically reduced proliferation in both cell lines. In contrast, a negligible impact was observed upon expression of the inactive RCE1 isoform 2 K43R mutant. However, expression of wild-type RCE1 isoform 2 also markedly reduced proliferation to a level nearly equivalent to USP17 knockdown (Figure 7c, Supplemental Figure 8). These results indicated that the regulation of RCE1 isoform 2 would appear to play an important role in the impact of USP17 upon cell growth.

\section{DISCUSSION}

Previously, our studies had indicated that USP17 could regulate RCE1 activity, potentially by deubiquitinating it [11]. However, no change was observed in the stability of RCE1 and it was unclear exactly how USP17 acted to regulate its activity, leading us to investigate further how this regulation was achieved. As a result of these studies we have now made a number of novel observations. Firstly, we have identified an additional RCE1 isoform (isoform 2) which is conserved in a number of mammalian species and is necessary, in tandem with RCE1 isoform 1, to reconstitute H-Ras plasma membrane localisation in RCE1-/- MEF cells. In addition, we have also shown that RCE1 isoform 2 is ubiquitinated on lysine 43 and that its localisation to the ER is dependent upon this ubiquitination. Finally, we have shown that USP17 can deubiquitinate lysine 43 and regulate the localisation and stability of isoform 2, and that isoform 2 over-expression can counteract the impact of USP17 on HRas localisation.

The observation that a second human transcript for RCE1 had been annotated within the RCE1 gene record led us to investigate if it represented a genuine transcript or if it was a database anomaly. The observation of equivalent transcripts in four other mammalian species and the presence of both transcripts in a range of human cell lines indicated that it was indeed 'bona fide'. This was further supported by the observation that USP17 could regulate the stability and localisation of 
isoform 2 specifically, and that its over-expression could counteract the action of USP17 on H-Ras localisation. Final confirmation came when we demonstrated that both RCE1 isoform 1 and isoform 2 were necessary to reconstitute H-Ras plasma membrane localisation in RCE1-/- MEF cells.

The necessity for this additional RCE1 transcript to reconstitute H-Ras plasma membrane localisation in RCE1-/- MEF cells was surprising as the previously recognised human RCE1 transcript had been shown to reconstitute RCE1 activity in S. cerevisiae [10] suggesting it has all the necessary residues for activity. However, it is interesting to note that we have only identified transcripts for RCE1 isoform 2 in mammalian species, that the USP17 orthologues are restricted to mammals [27], and that lysine 43 (lysine 147 in isoform 1), which is required for the ubiquitination of isoform 2, is also apparently restricted to vertebrate species (Supplemental Figure 9). This would suggest mammalian cells have evolved an additional role for RCE1 which is mediated via the production and regulation of isoform 2 .

It is also unclear how isoform 2 contributes to H-Ras plasma membrane localisation. Isoform 1 and isoform 2 are both localised to the ER, but it is not known if they interact and it is unclear if they act together, or if they have independent roles. Isoform 2 could be a co-factor required for the activation of isoform 1, or it could act in conjunction with isoform 1 to elicit RCE1 activity (Figure 8a). Alternatively, isoform 2 could play an entirely different, and as yet unidentified, role in this processing pathway which is independent of isoform 1 (Figure $8 \mathrm{~b}$ ). This job could be facilitated by other proteins in non-mammalian cells, or might not be required in these cells. However, whatever the role of isoform 2, additional studies will be required to elucidate this further.

In regards to the regulation of RCE1 isoform 2 by ubiquitination and USP17, it is clearly evident that ubiquitin chains are added to lysine 43 of isoform 2 and that these can be removed by USP17. The addition of ubiquitin would also appear to allow the retention of isoform 2 within the ER as both its removal by USP17, and the loss of lysine 43, result in the re-localisation of isoform 2 away from the ER (Figure 8c). This could also explain the impact of USP17 on H-Ras localisation because whatever role isoform 2 plays in regards to H-Ras might only be accomplished when it is localised to the ER. How the ubiquitination of isoform 2 retains it within the ER is not clear. It could allow its retention due to the ubiquitinated protein being able to interact with other ER resident proteins, or alternatively it could block the binding of proteins which remove it from the ER.

Previously we had hypothesised that RCE1 isoform 1 was ubiquitinated [11], but this hypothesis now requires revision in light of our discovery of RCE1 isoform 2 . These original observations were probably due to the difficulties of immuno-precipitating a membrane integral protein such as isoform 1 without isolating associated membrane proteins such as isoform 2. However, our data now demonstrates that it is RCE1 isoform 2 that is ubiquitinated and that lysine 43 is crucial for this ubiquitination as well as the regulation of isoform 2 by USP17. This new hypothesis is further supported by the observation that an isoform 1 mutant lacking lysine residues is still able to contribute to the plasma membrane localisation of H-Ras in RCE1 -/- MEF cells suggesting isoform 1 does not require ubiquitination to function.

Interestingly, our data also highlighted that USP17 modulated the stability of isoform 2, but in a counter-intuitive manner. If RCE1 isoform 2 was being targeted to the proteasome you would have expected that its deubiquitination by USP17 would rescue it, rather than leading to its degradation. However, we have also shown that RCE1 isoform 2 is ubiquitinated with K63-linked chains which are not normally associated with proteasomal degradation. Furthermore, it is interesting to note that mutating lysine 43 from RCE1 isoform 2 didn't impact upon its stability. We now hypothesise that the ubiquitination of isoform 2 localises it to the ER, and once this is removed by USP17, isoform 2 leaves the ER and is subsequently degraded, thus blocking the processing of ' $\mathrm{CaaX}$ ' motif proteins (Figure 8). In addition, it could be speculated that upon the transient induction of USP17 transcription in response to a range of stimuli [17, 23], or during the cell cycle [22], that the level of isoform 2, and thus the processing of ' $\mathrm{CaaX}$ ' motif proteins, is similarly modulated. This hypothesis will however require further investigation to confirm its validity.

A number of substrates have been proposed for USP17 such as Cdc25A [21], SDS3 [26] and now RCE1 isoform 2 and it is unclear how significant each of these proposed substrates is in regards to the action of USP17. However, our previous studies demonstrated that USP17 did not impact upon 
cell growth in the absence of RCE1 [11] and our data here would suggest that RCE1 isoform 2 is a major substrate for USP17, particularly in regards to its impact upon cell proliferation.

In summary, we have shown that RCE1 has an additional isoform in a range of mammalian species which is required for the reconstitution of H-Ras localisation in RCE1-/- MEF cells. In addition, we have shown that RCE1 isoform 2 is ubiquitinated on $\mathrm{K} 43$ and that this ubiquitination retains it within the ER. Also, we have demonstrated that USP17 can remove this ubiquitination and re-localise isoform 2 out of the ER, and that isoform 2 appears to be a major USP17 substrate, as its over-expression results in a block in cell growth. These observations may not only be significant to our understanding of how RCE1 and USP17 function, but may also aid in any future drug discovery efforts to target either USP17 or RCE1.

\section{REFERENCES}

1] Reiss, Y., Goldstein, J.L., Seabra, M.C., Casey, P.J., Brown, M.S. (1990) Inhibition of purified p21ras farnesyl protein transferase by Cys-AAX tetrapeptides. Cell 62, 81-88

2] Boyartchuk, V.L., Ashby, M.N., Rine, J. (1997) Modulation of Ras and a-factor function by carboxyl-terminal proteolysis. Science 275, 1796-1800

3] Kim, E., Ambroziak, P., Otto, J.C., Taylor, B., Ashby, M., Shannon, K., Casey, P.J., Young, S.G. (1999) Disruption of the mouse Rcel gene results in defective Ras processing and mislocalization of Ras within cells. J Biol. Chem. 274, 8383-8390

4] Otto, J.C., Kim, E., Young, S.G., Casey, P.J. (1999) Cloning and characterization of a mammalian prenyl protein-specific protease. J Biol. Chem. 274, 8379-8382

5] Hrycyna, C.A., Sapperstein, S.K., Clarke, S., Michaelis, S. (1991) The Saccharomyces cerevisiae STE14 gene encodes a methyltransferase that mediates C-terminal methylation of a-factor and RAS proteins. EMBO J. 10, 1699-1709

6] Choy, E., Chiu, V.K., Silletti, J., Feoktistov, M., Morimoto, T., Michaelson, D., Ivanov, I.E., Philips, M.R. (1999) Endomembrane trafficking of ras: the CAAX motif targets proteins to the ER and Golgi. Cell 98, 69-80

7] Apolloni, A., Prior, I.A., Lindsay, M., Parton, R.G., Hancock, J.F. (2000) H-ras but not K-ras traffics to the plasma membrane through the exocytic pathway. Mol. Cell. Biol. 20, 2475-2487 8] Roberts, P.J., Mitin, N., Keller, P.J., Chenette, E.J., Madigan, J.P., Currin, R.O., Cox, A.D., Wilson, O., Kirschmeier, P., Der, C.J. (2008) Rho Family GTPase modification and dependence on CAAX motif-signaled posttranslational modification. J. Biol. Chem. 283, 25150-25163

9] Dolence, J.M., Steward, L.E., Dolence, E.K., Wong, D.H., Poulter, C.D. (2000) Studies with recombinant Saccharomyces cerevisiae CaaX prenyl protease Rcelp. Biochemistry 39, 4096-104 10] Plummer, L.J., Hildebrandt, E.R., Porter, S.B., Rogers, V.A., McCracken, J., Schmidt, W.K. (2006) Mutational analysis of the ras converting enzyme reveals a requirement for glutamate and histidine residues. J Biol. Chem. 281, 4596

11] Burrows, J.F., Kelvin, A.A., McFarlane, C., Burden, R.E., McGrattan, M.J., de la Vega, M., Govender, U., Quinn, D.J., Dib, K., Gadina, M., Scott, C.J., Johnston, J.A. (2009) USP17 regulates Ras activation and cell proliferation by blocking RCE1 activity. J Biol Chem. 284, 9587-9595

12] Kumar, A., Beresini, M.H., Dhawan, P., Mehta, K.D. (1996) Alpha-subunit of farnesyltransferase is phosphorylated in vivo: effect of protein phosphatase-1 on enzymatic activity. Biochem. Biophys. Res. Commun. 222, 445-452

13] Kumar, A., Mehta, K.D. (1997) p21ras farnesyltransferase alpha- and beta-subunits are phosphorylated in PC-12 cells: TGF-beta signaling pathway independent phosphorylation. Neurosci. Lett. 231, 143-146

14] Goalstone, M., Carel, K., Leitner, J.W., Draznin, B. (1997) Insulin stimulates the phosphorylation and activity of farnesyltransferase via the Ras-mitogen-activated protein kinase pathway.

Endocrinology 138, 5119-5124

15] Zhu, Y., Os Pless, M., Inhorn, R., Mathey-Prevot, B., D’Andrea, A.D. (1996) The murine DUB-1 gene is specifically induced by the betac subunit of interleukin-3 receptor. Mol. Cell. Biol. 16, 48084819 
16] Zhu, Y., Lambert, K., Corless, C., Copeland, N.G., Gilbert, D.J., Jenkins, N.A., D'Andrea, A.D. (1997) DUB-2 is a member of a novel family of cytokine-inducible deubiquitinating enzymes. J Biol. Chem. 272, 51-57

17] Burrows, J.F., McGrattan, M.J., Rascle, A., Humbert, M., Baek, K.H., Johnston, J.A. (2004)

DUB-3, a cytokine-inducible deubiquitinating enzyme that blocks proliferation. J Biol. Chem. 279, 13993-14000

18] Zhu, Y., Carroll, M., Papa, F.R., HochStrasser, M., D’Andrea, A. (1996) DUB-1, a deubiquitinating enzyme with growth-suppressing activity. Proc. Natl. Acad. Sci. USA 93, 3275-3279

19] Migone, T., Humbert, M., Rascle, A., Sanden, D., D'Andrea, A., Johnston, J.A. (2001) The deubiquitinating enzyme DUB-2 prolongs cytokine-induced signal transducers and activators of transcription activation and suppresses apoptosis following cytokine withdrawal. Blood 98, 19351941

20] Shin, J.M., Yoo, K.J., Kim, M.S., Kim, D., Baek, K.H. (2006) Hyaluronan- and RNA-binding deubiquitinating enzymes of USP17 family members associated with cell viability. BMC Genomics 7 , 292

21] Pereg, Y., Liu, B.Y., O’Rourke, K.M., Sagolla, M., Dey, A., Komuves, L., French, D.M., Dixit, V.M. (2010) Ubiquitin hydrolase Dub3 promotes oncogenic transformation by stabilizing Cdc25A. Nature Cell Biology 12, 400-406

22] McFarlane, C., Kelvin, A.A., de la Vega, M.A., Govender, U., Scott, C.J., Burrows, J.F., Johnston, J.A. (2010) The deubiquitinating enzyme USP17 is cell cycle regulated and mediates G1-S transition. Cancer Res. 70, 3329-3339

23] de la Vega, M., Kelvin, A.A., Dunican, D.J., McFarlane, C., Burrows, J.F., Jaworski, J., Stevenson, N.J., Dib, K., Rappoport, J.Z., Scott, C.J., Long, A., Johnston, J.A. (2011) The deubiquitinating enzyme USP17 is essential for GTPase subcellular localization and cell motility. Nat Commun. 2, 259

24] de la Vega, M.A., Burrows, J.F., McFarlane, C., Govender, U., Scott, C.J., Johnston, J.A. (2010) The deubiquitinating enzyme USP17 blocks N-Ras membrane trafficking and activation, but leaves K-Ras unaffected. J Biol Chem. 285, 12028-12036 25] Schmidt, W.K., Tam, A., Fujimura-Kamada, K., Michaelis, S. (1998) Endoplasmic reticulum membrane localization of Rce1p and Ste24p, yeast proteases involved in carboxyl-terminal CAAX protein processing and amino-terminal a-factor cleavage. Proc Natl Acad Sci U S A. 95, 11175-11180 26] Ramakrishna, S., Suresh, B., Lee, E.J., Lee, H.J., Ahn, W.S., Baek, K.H. (2011) Lys-63-specific deubiquitination of SDS3 by USP17 regulates HDAC activity. J Biol Chem. 286, 10505-10514 27] Burrows, J.F., Scott, C.J., Johnston, J.A. (2010) The DUB/USP17 deubiquitinating enzymes: a gene family within a tandemly repeated sequence, is also embedded within the copy number variable beta-defensin cluster. BMC Genomics.11, 250

\section{ACKNOWLEDGEMENTS}

We thank the members of the Burrows, Scott and Johnston labs for their technical assistance. We also thank Dr Derek Quinn (Queen's University, Belfast, UK) for his gift of plasmids.

\section{FUNDING}

This study was supported by a grant from the Biotechnology and Biological Sciences Research Council (BBSRC) (Grant number: BB/F013647/1). 


\section{Figure Legends}

Figure 1: There are two RCE1 isoforms. A) Two transcripts for human RCE1 have been identified in the databases. Isoform 2 lacks 4 bases due to the use of an alternative splice site and as a result initiates its ORF at methionine 105 of isoform 1. B) RNA samples were harvested from 293T (fibroblast), H460 (lung) and HCT116 (colorectal) cells and RT-PCR was carried out using primers specific for RCE1 isoforms 1 and 2 as well as a beta-actin primer set to control for loading. C) 293T cells were transiently transfected with vectors expressing RCE1-ISO1-FLAG and RCE1-ISO2-FLAG. Lysates were extracted and immuno-precipitated where indicated with the M2 antibody and immunoblotted for FLAG epitope tagged RCE1.

Figure 2: USP17 regulates RCE1 isoform 2. A) 293T cells were transfected with an expression construct for RCE1-ISO2-FLAG in conjunction with either USP17 or USP17CS. The resulting lysates were assessed by immuno-blotting with either an anti-USP17, anti-FLAG or anti-tubulin antibody. B) 293T cells were transfected with RCE1-ISO2-FLAG in conjunction with either a scrambled shRNA, or one of two USP17 specific shRNAs. The resulting lysates were assessed by immuno-blotting with either an anti-FLAG or anti-tubulin antibody. USP17 knockdown was also confirmed by RT-PCR using USP17 and GAPDH specific primer sets. C) 293T cells were transfected with RCE1-ISO2-HIS and HA-ubiquitin in conjunction with either USP17, or USP17CS. The resulting lysates were precipitated using IMAC beads and the precipitates and lysates were assessed by immuno-blotting with either an anti-HA, anti-HIS, anti-USP17 or anti-tubulin antibody. D) Hela cells were transfected with RCE1-ISO1-FLAG or RCE1-ISO2-FLAG in conjunction with either USP17 or USP17CS and the cells were fixed and stained using antibodies against FLAG (red) and the ER marker calnexin (green). The vectors used in each panel are as indicated and the scale bars are $10 \mu \mathrm{M}$.

\section{Figure 3: RCE1 isoform 2 blocks USP17 regulation of GFP-H-Ras localisation}

A) Hela cells were transfected with combinations of RCE1-ISO2-FLAG, GFP-H-Ras, USP17 and USP17CS and the cells were fixed and stained for USP17 before being visualised using a confocal microscope. Scale bars are $20 \mu \mathrm{M}$. B) The cells from each chamber were assessed for the presence of USP17 and then the localisation of GFP-H-Ras was classed as being either mainly plasma membrane or mainly cytosolic. The first 100 cells were assessed and the pooled results of two experiments are illustrated as a bar chart showing the percentage of cells with mainly plasma membrane localisation of GFP-H-Ras.

Figure 4: RCE1 isoform 1 and 2 are required to re-localise $H$-Ras to the plasma membrane in RCE1-/- MEF cells. A) Wild-type and RCE1-/- MEF cells were transfected with GFP-H-Ras and combinations of empty vector, RCE1-ISO1-FLAG or RCE1-ISO2-FLAG as indicated. The cells were fixed and the nuclei stained with DAPI prior to being assessed using confocal microscopy. The lower panels are enlarged images of the indicated area in the upper panels. Scale bar are $10 \mu \mathrm{m}$. B) The cells from each chamber were examined and the localisation of GFP-H-Ras was classed as being either mainly plasma membrane or mainly cytosolic. The first 100 cells were assessed and the pooled results of two experiments are illustrated as a bar chart showing the percentage of cells with mainly plasma membrane localisation of GFP-H-Ras.

Figure 5: Lysine residues are only required in RCE1 isoform 2 to re-localise H-Ras to the plasma membrane in RCE1-/- MEF cells. A) RCE1-/- MEF cells were transfected with GFP-H-Ras and combinations of RCE1-ISO1-FLAG, RCE1-ISO2-FLAG, or the mutants RCE1-ISO1-4KRFLAG and RCE1-ISO2-2KR-FLAG which lack lysine residues, as indicated. The cells were fixed and the nuclei stained with DAPI prior to being assessed using confocal microscopy. The lower panels are enlarged images of the indicated area in the upper panels. Scale bars are $10 \mu \mathrm{m}$. B) The cells from each chamber were examined and the localisation of GFP-H-Ras was classed as being either mainly plasma membrane or mainly cytosolic. The first 100 cells were assessed and the pooled results of two experiments are illustrated as a bar chart showing the percentage of cells with mainly plasma membrane localisation of GFP-H-Ras. 
Figure 6: Lysine 43 is required for RCE1 isoform 2 ubiquitination and to re-localise H-Ras to the plasma membrane in RCE1-/- MEF cells. A) RCE1-/- MEF cells were transfected with GFP-HRas and combinations of RCE1-ISO1-FLAG, RCE1-ISO2-FLAG, or the mutants RCE1-ISO2-K43RFLAG and RCE1-ISO2-K202R-FLAG, as indicated. The cells were fixed and the nuclei stained with DAPI prior to being assessed using confocal microscopy. The lower panels are enlarged images of the indicated area in the upper panels. Scale bars are $10 \mu \mathrm{m}$. B) The cells from each chamber were examined and the localisation of GFP-H-Ras was classed as being either mainly plasma membrane or mainly cytosolic. The first 100 cells were assessed and the pooled results of two experiments are illustrated as a bar chart showing the percentage of cells with mainly plasma membrane localisation of GFP-H-Ras. C) 293T cells were transfected with combinations of HA-ubiquitin and either RCE1ISO2-HIS, RCE1-ISO2-K43R-HIS or RCE1-ISO2-K202R-HIS. The resulting lysates were precipitated using IMAC beads and the precipitates and lysates were assessed by immuno-blotting with either an anti-HA, anti-HIS or anti-tubulin antibody.

Figure 7: K43 is required for RCE1 isoform 2 ER localisation and regulation by USP17 A) Hela cells were transfected with RCE1-ISO2-K43R-FLAG or RCE1-ISO2-K202R-FLAG in conjunction with either USP17 or USP17CS and the cells were fixed and stained using antibodies against FLAG (red) and the ER marker calnexin (green). The vectors used in each panel are as indicated, the lower panels are enlarged images of the indicated area in the upper panels and the scale bars are $10 \mu \mathrm{m}$. B) 293T cells were transiently transfected with RCE1-ISO2-FLAG, RCE1-ISO2K43R or RCE1-ISO2-K202R-FLAG in conjunction with empty vector, USP17 or USP17CS. Lysates were extracted and immuno-blotted using anti-FLAG, anti-USP17 and anti-tubulin antibodies as indicated. C) Hela cells were transfected with empty vector, RCE1-ISO2-FLAG, RCE1-ISO2-K43RFLAG, scrambled shRNA or USP17shRNA1. An MTT assay was then performed on samples taken after the time-points specified.

Figure 8: The role of USP17 and RCE1 isoform 2 in 'CaaX' motif processing We have two possible hypotheses in regards to the function of RCE1 isoform 2 in the processing of 'CaaX' proteins. A) Upon farnestylation H-Ras associates with the ER and RCE1 isoform 1 (ISO1) and isoform 2 (ISO2) act as a complex to allow the proper cleavage of the H-Ras 'CaaX' motif and thus its subsequent processing and plasma membrane localisation. B) Upon farnestylation H-Ras associates with the ER and RCE1 isoform 1 (ISO1) cleaves the H-Ras 'CaaX' motif. Isoform 2 is then involved in a subsequent, and currently unidentified, processing step prior to H-Ras plasma membrane localisation. C) RCE1 isoform 2 is retained within the ER when it is ubiquitinated on lysine 43 with K63-linked ubiquitin chains (Panel 1). When USP17 is expressed it removes the K63-linked ubiquitin chains from lysine 43 of RCE1 isoform 2 and this blocks the processing of the 'CaaX' motif of H-Ras (Panel 2). The removal of the K63-linked ubiquitin chains from K43 of isoform 2 results in its relocalisation out of the ER and its degradation (Panel 3). 